\title{
Degeneration of spinal motor neurons by chronic AMPA-induced excitotoxicity in vivo and protection by energy substrates
}

Citlalli Netzahualcoyotzi and Ricardo Tapia*

\begin{abstract}
Introduction: Several data suggest that excitotoxicity due to excessive glutamatergic neurotransmission may be an important factor in the mechanisms of motor neuron (MN) death occurring in amyotrophic lateral sclerosis (ALS). We have previously shown that the overactivation of the $\mathrm{Ca}^{2+}$-permeable a-amino-3-hydroxy-5-methyl-4-isoxazole propionate (AMPA) glutamate receptor type, through the continuous infusion of AMPA in the lumbar spinal cord of adult rats during several days, results in progressive rear limb paralysis and bilateral MN degeneration. Because it has been shown that energy failure and oxidative stress are involved in MN degeneration, in both ALS and experimental models of spinal MN degeneration, including excitotoxicity, in this work we tested the protective effect of the energy substrates pyruvate and $\beta$-hydroxybutyrate $(\beta H B)$ and the antioxidants glutathione ethyl ester (GEE) and ascorbate in this chronic AMPA-induced neurodegeneration.

Results: AMPA infusion induced remarkable progressive motor deficits, assessed by two motor tasks, that by day seven reach bilateral rear limb paralysis. These effects correlate with the death of $>80 \%$ of lumbar spinal MNs in the infused and the neighbor spinal cord segments, as well as with notable astrogliosis in the ventral horns, detected by glial fibrillary acidic protein immunohistochemistry. Co-infusion with pyruvate or $\beta \mathrm{HB}$ notably prevented the motor deficits and paralysis, decreased MN loss to $<25 \%$ and completely prevented the induction of astrogliosis. In contrast, the antioxidants tested were ineffective regarding all parameters analyzed.

Conclusions: Chronic progressive excitotoxicity due to AMPA receptors overactivation results in MN death and astrogliosis, with consequent motor deficits and paralysis. Because of the notable protection against these effects exerted by pyruvate and $\beta \mathrm{HB}$, which are well established mitochondrial energy substrates, we conclude that deficits in mitochondrial energy metabolism are an important factor in the mechanisms of this slowly developed excitotoxic MN death, while the lack of protective effect of the antioxidants indicates that oxidative stress seems to be less significant factor. Because excitotoxicity may be involved in MN degeneration in ALS, these findings suggest possible preventive or therapeutic strategies for the disease.
\end{abstract}

Keywords: AMPA, Amyotrophic lateral sclerosis, Antioxidants, Energy substrates, Excitotoxicity, Motor neurons, Neurodegeneration, Spinal cord

\section{Introduction}

Amyotrophic lateral sclerosis (ALS) is a lethal neurodegenerative disorder characterized by the death of the upper motor neurons (MNs) in the motor cortex and the lower ones in the brain stem and the spinal cord. ALS patients develop a progressive muscle paralysis and there is still no

\footnotetext{
* Correspondence: rtapia@ifc.unam.mx

División de Neurociencias, Instituto de Fisiología Celular, Universidad Nacional Autónoma de México, AP 70-253, 04510 México DF, México
}

effective treatment. About 10\% of ALS cases involve genetic alterations and are therefore termed familial ALS, but the etiology of the remainder 90\%, sporadic ALS, is still largely unknown. Among the many factors that have been involved in the mechanisms of MN degeneration are glutamate receptor-mediated excitotoxicity, oxidative stress, mitochondrial dysfunction, neuroinflammation, immune-mediated processes, protein aggregation and axonal transport alterations $[1,2]$, but there is still no satisfactory explanation of why such factors damage predominantly MNs. 
Mitochondrial oxidative metabolism seems to be involved in ALS pathogenesis, since morphological and functional alterations in mitochondria of spinal MNs and skeletal muscle of ALS patients have been described [3-6], as well as mitochondrial swelling and vacuolization in the spinal cord of transgenic mutant superoxide dismutase 1 (SOD1) ALS mice [7,8]. In addition, isolated mitochondria from the spinal cord of these mutant SOD1 mice showed decreased $\mathrm{Ca}^{2+}$ buffering capacity [8], and dysfunction of oxidative phosphorylation and consequently decreased ATP synthesis $[9,10]$.

The excitotoxic process involves the entrance of $\mathrm{Ca}^{2+}$ through glutamate receptors, and the resulting disruption of $\mathrm{Ca}^{2+}$ homeostasis activates lytic enzymes, induces the overproduction of reactive oxygen species and leads to mitochondrial dysfunction and energy failure [2,11-13]. Work from our laboratory has shown that such excitotoxic mechanism, mediated mainly by overactivation of the $\mathrm{Ca}^{2+}$-permeable AMPA ( $\alpha$-amino-3-hydroxy-5-methylisoxazole-4-propionate)-type receptors induces the death of spinal MNs in the rat in vivo and that this results in progressive paralysis. Acute or chronic infusion of AMPA in the lumbar spinal cord, by means of microdialysis $[14,15]$ or osmotic minipumps [16], respectively, results in MN degeneration that correlated with motor alterations and finally paralysis of the rear limbs, in 3-12 $\mathrm{h}$ after microdialysis or in 3-5 days after the implantation of the osmotic pump. The acute MN death and ipsilateral paralysis caused by AMPA microdialysis perfusion was prevented or reduced by specific $\mathrm{Ca}^{2+}$-permeable AMPA receptor antagonists and by intracellular $\mathrm{Ca}^{2+}$ chelators [15,17], demonstrating the vulnerability of spinal MNs to the excitotoxic effect of $\mathrm{Ca}^{2+}$-permeable AMPA receptors activation in vivo, which had been previously reported only in studies in neuronal cultures in vitro [18-20]. Using the acute microdialysis procedure, which results in rapid ipsilateral $\mathrm{MN}$ loss and motor alterations of the corresponding rearlimb, we recently showed that among several energy substrates and antioxidants tested, pyruvate and $\beta$ hydroxybutyrate $(\beta \mathrm{HB})$ notably protected against the excitotoxic effects of AMPA whereas the antioxidants tested were ineffective. These results allowed us to conclude that failure in mitochondrial energy metabolism was greatly involved in this rapid MN death, and also permitted to establish a correlation between the number of dead MNs and the degree of unilateral motor alterations [21]. Nonetheless, in this microdialysis acute experiment AMPA is perfused during $25 \mathrm{~min}$ and produces only unilateral paralysis, MN alterations and motor deficits that develop in less than $6 \mathrm{~h}$, whereas the chronic slow continuous administration of AMPA through osmotic minipumps induces bilateral rear limb paralysis that progresses slowly along several days, also correlated with MN loss but extended to both ventral horns. Therefore, we considered of interest to evaluate the efficacy of the energy substrates and the antioxidants to protect against the chronic AMPA excitotoxic action. In addition, we correlated the protective effect along the progress of motor alterations with the MN loss in the infused region and in the immediate rostral region of the lumbar spinal cord, as well as with the prevention of astrogliosis induced by AMPA.

\section{Materials and methods}

Adult male Wistar rats (280-300 g) were used in all experiments and were handled in accordance with international standards of animal welfare and with approval of the Institutional Committee for the Care and Use of Laboratory Animals (Approval No. RTI21-14). Rats were housed in a laboratory environment with a $12 \mathrm{~h}$ light/ dark cycle and with food and water ad libitum. Animals showing complete paralysis and loss of the righting reflex within $15 \mathrm{~s}$ were sacrificed to avoid unnecessary suffering and were not analyzed further.

\section{Osmotic minipumps implantation}

The implantation of the osmotic minipumps in the lumbar spinal cord was carried out essentially as previously described [16] with the following modifications.

All drugs were dissolved in $0.1 \mathrm{M}$ phosphate buffer, $\mathrm{pH}$ 7.4. After some preliminary experiments with 7.5, 4.5, 3.5 and $1 \mathrm{mM}$ AMPA (Tocris, Ellisville, MO, USA), the latter concentration was chosen because it induced a slow and progressive paralysis of the rear limbs along several days, whereas the effect of the higher doses was more rapid and severe. We tested the effect of the energy substrates pyruvate and $\beta \mathrm{HB}$, and the antioxidants ascorbate and glutathione ethyl ester (GEE) (SigmaAldrich, Saint Louis, MO, USA) at concentration of $20 \mathrm{mM}$, based on our previous work with AMPA acute administration [21]. GEE was used instead of glutathione because it enters the cell more efficiently [22]. Osmotic minipumps (Alzet model 2004, capacity $\sim 250 \mu \mathrm{L}$, flow rate $6 \mu \mathrm{l} /$ day) were filled with one of the following solutions: vehicle as control, $1 \mathrm{mM}$ AMPA, AMPA + sodium pyruvate, AMPA + DL- $\beta$ HB sodium salt, AMPA + GEE, AMPA + L-ascorbic acid, and AMPA + antioxidant mixture (GEE + ascorbate), and they were incubated in sterile saline solution at $37^{\circ} \mathrm{C}$ for $48 \mathrm{~h}$ for stabilization before the implantation. The day of surgery rats were anesthetized with isoflurane $1-2 \%$ in a $95 \% \mathrm{O}_{2}+5 \% \mathrm{CO}_{2}$ mixture and placed in a stereotaxic spinal unit. A longitudinal incision of the skin was made in the lumbar region and muscles surrounding the lumbar vertebrae were retracted. A stainless-steel screw $(3.7 \mathrm{~mm}$ long, $1 \mathrm{~mm}$ diameter) was inserted on the left side of the third lumbar vertebra without reaching the surface of the spinal cord to anchor the implant. A 1-2 mm diameter hole was drilled at the same vertebrae and a small cut of 
the meninges was made to insert a borosilicate glass probe (1 mm long, 0.5 I.D. x 0.8 O.D., VitroCom Inc.) into the right dorsal horn of the spinal cord. The other end of the cannula was connected through a plastic tube to the osmotic minipump which was placed subcutaneously in the back of the rat, caudally to the skin incision. The probe and the screw were fixed to the bone with dental acrylic. At the end of the surgery, the skin incision was closed with surgical stainless-steel clips and rats received a single i.m. dose of penicillin (50 U). After recovering from anesthesia animals were kept in individual cages with water and food ad libitum until perfusion for histological analysis. Neither the control nor the experimental animals showed signs of pain or suffering during the time period studied, even during their performance in the motor tests.

\section{Assessment of motor function}

Before surgery rats were trained to achieve $120 \mathrm{~s}$ on the rotarod test (Columbus Instruments), in which rotation started at $10 \mathrm{rpm}$ and accelerated at $0.2 \mathrm{rpm} / \mathrm{s}$, and also to perform a PGE task, where the latency to climb a vertical grid is scored. Three trials/day/rat were assessed in each motor test. In addition, the hind paw footprint was registered using non-toxic Chinese ink on the hind paws of rats. Besides, holding up the tail we photographically recorded the changes in the position of the rear limbs. These motor evaluations were assessed daily five days prior surgery until the seventh day, when animals were anesthetized and perfused for histology.

\section{Histology and immunohistochemistry}

Seven days after osmotic pump implantation rats were perfused and fixed for histological and immunohistochemical analyses as previously described [16]. Briefly, spinal cords were removed, postfixed $48 \mathrm{~h}$ at $4^{\circ} \mathrm{C}$ and dehydrated with $20 \%$ and $30 \%$ sucrose solutions. Transverse sections ( $45 \mu \mathrm{m}$ thick) of the spinal cords were obtained in a cryostat. Fifty serial slices at the site of the cannula infusion (infused region) and the next fifty rostral slices (rostral region) were obtained, so that the total tissue analyzed was $2.25 \mathrm{~mm}$ length, about 2 lumbar segments each region. Alternate slices were stained with cresyl violet or immunostained for neurofilament protein SMI-32 and glial fibrillary acidic protein (GFAP). The number of morphologically healthy MNs (i.e. large neurons with a soma diameter $>20 \mu \mathrm{m}$ and distinguishable nucleus, similar in appearance to those of the control and intact rats) in both the ipsilateral and contralateral ventral horns were counted in fifteen Nissl-stained slices/rat/region and the values were averaged.

For immunohistochemistry, mouse polyclonal antiSMI-32 (1:500, Abcam), chicken anti-GFAP (1:1000, Abcam), as markers of neurons and astrocytes, respectively;
Texas-red-conjugated anti-mouse (1:200, Invitrogen) and fluorescein-conjugated anti-chicken (1:200, Novex) antibodies were used. Sections were mounted on silanecovered slides and coverslipped with fluorescent mounting medium (DAKO). Sections were visualized under epifluorescent microscopy and merged images of the overlay of FITC and Texas Red channels were obtained with Image J (NIH, Bethesda, USA). The cross-reactivity in the immunofluorescent technique was excluded by control slices incubated in the absence of primary antibodies. There was no immunostaining in these controls.

\section{Statistics}

The statistical analysis of the number of healthy MNs, the rotarod and PGE performance was carried out using ANOVA followed by a Tukey's post hoc test. A value of $\mathrm{p}<0.05$ was considered statistically significant.

\section{Results}

\section{Energy substrates exert better protection than} antioxidants against the AMPA-induced motor alterations None of the control rats showed motor dysfunctions (Figures 1 and 2) or histological damage (Figures 3, 4 and 5) at any time up to 7 days, the maximum period studied. Perfusion of pyruvate, $\beta \mathrm{HB}, \mathrm{GEE}$ or ascorbate alone at $20 \mathrm{mM}$ concentration ( $\mathrm{n}=3$ per group) was also innocuous (not shown). The chronic $7.5 \mathrm{mM}$ AMPA infusion in the lumbar spinal cord of rats induced a gradual paralysis of the rear limbs in about 3 days [16]. As mentioned in Methods, in this work we reduced the concentration from $7.5 \mathrm{mM}$ to $1 \mathrm{mM}$ in order to extend the time of progression of symptoms. AMPA induced a paralysis that began in the distal ipsilateral rear limb (phalanges), ascending through the tarsus until the entire limb; the same process occurred with a short delay in the contralateral limb. This progression is shown in Figure 1 at days 1, 4 and 7. Quantitative assessment of these motor alterations in the rotarod and the paw grip endurance (PGE) test is shown in Figure 2, with emphasis in the values at these days to show the correlation with the position of the paws at these times. Whereas control rats remained in the rotarod for the total test period of $120 \mathrm{~s}$, AMPA-infused animals fell at $73 \mathrm{~s}$ at day $1,31-41 \mathrm{~s}$ at days $2-5$ and $21 \mathrm{~s}$ at day 7 . In the PGE task, control rats climbed always within $1 \mathrm{~s}$ whereas AMPA-treated animals at day 1 took double time to climb and then this time increased progressively until $7 \mathrm{~s}$ at day 4 and $10 \mathrm{~s}$ at day 7 .

Perfusion of pyruvate and $\beta \mathrm{HB}$ partially prevented the AMPA-induced rear limbs paralysis. As shown in Figure 1, animals from these groups were able to partially control the ipsilateral phalanges and there was only a slight weakness in the contralateral ones, manifested by an incomplete tarsus footprint contralateral paw. Although the 


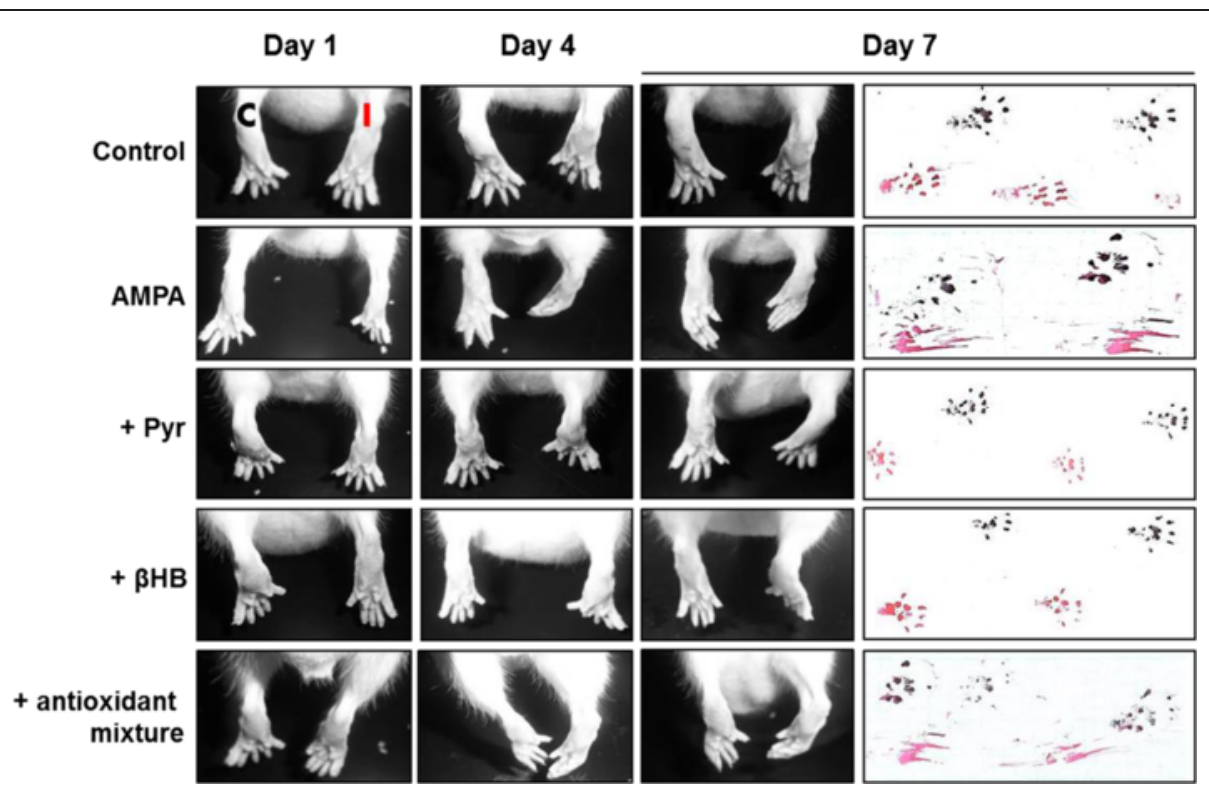

Figure 1 Progressive alterations of the rear limbs induced by AMPA and protection by energy substrates. Representative posterior views of the rear limbs (C, contralateral; I, ipsilateral) and hind paw footprints (red, ipsilateral; black, contralateral) at the initial (day 1), the middle (day 4) and last day (day 7) of treatment. The AMPA-induced progressive alterations were prevented by pyruvate and $\beta$ HB but not by ascorbate and GEE alone (not shown) or in combination (antioxidant mixture).

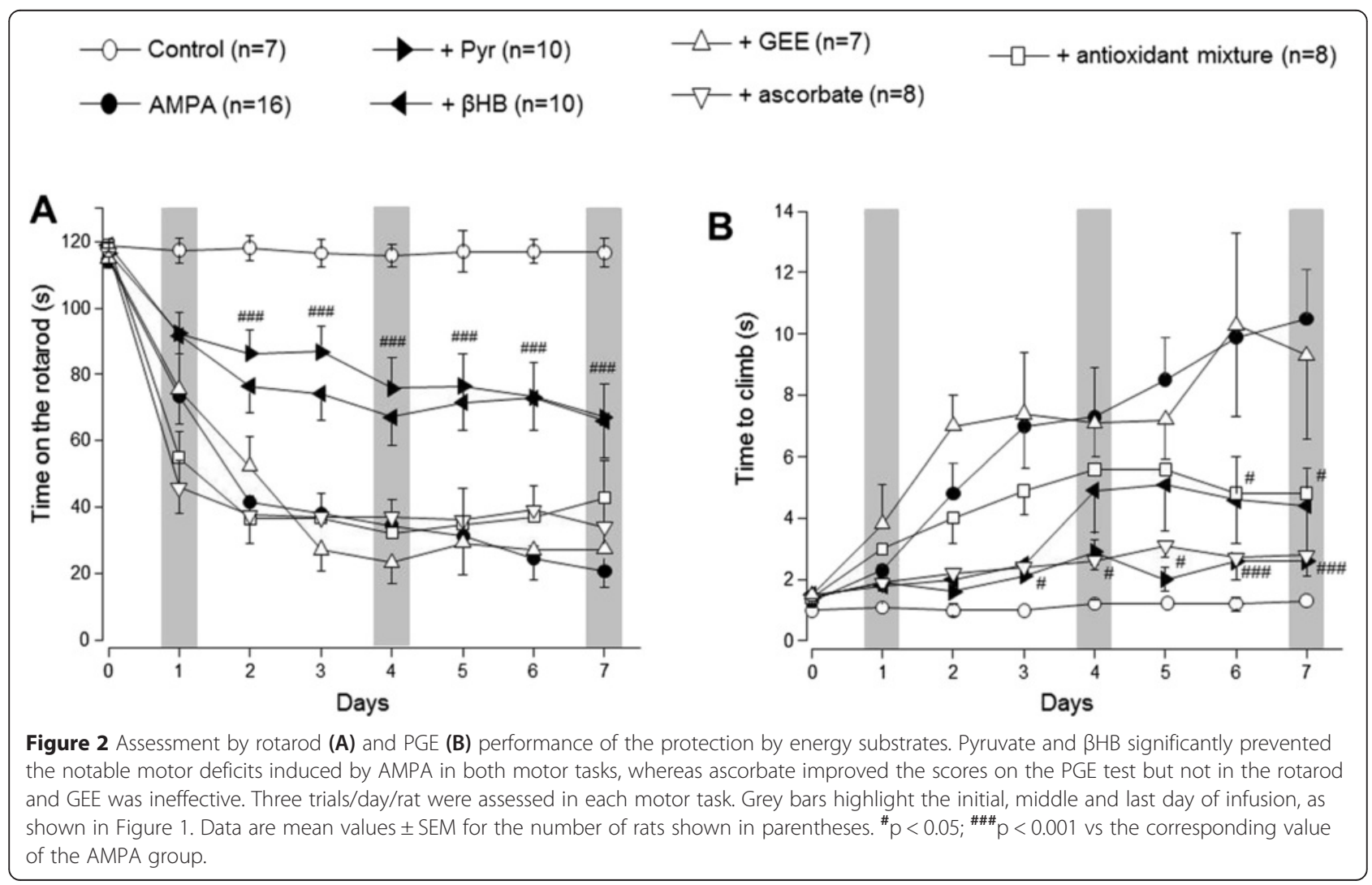




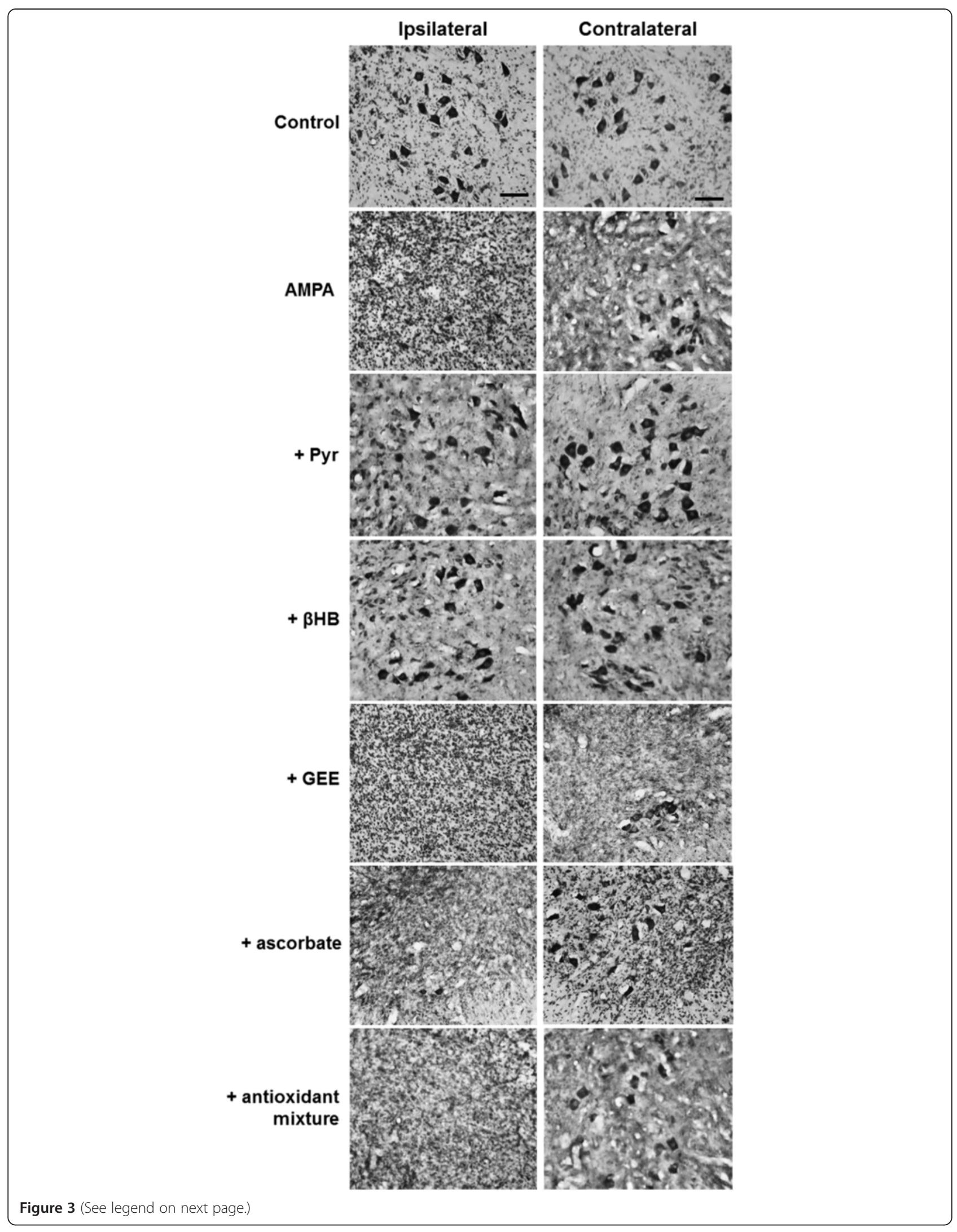


(See figure on previous page.)

Figure 3 AMPA-induced MN degeneration and protection by energy substrates. Representative micrographs of the ventral horns of Nissl-stained sections of the infused lumbar spinal cord region of rats treated as indicated, seven days after minipump implantation. AMPA induced an almost complete loss of MN in the infused side (ipsilateral) and a less intense degeneration in the contralateral side, and both effects were clearly prevented by pyruvate and $\beta$ HB but not by ascorbate or GEE. Quantitative analysis is shown in Figure 4 . Scale bar = $100 \mu m$.

energy substrates did not entirely prevent the above described AMPA-induced paralysis, its protective effect was highly significant, attaining $91 \mathrm{~s}$ in the rotarod performance at day 1 , and then 66-76 s from day 4 to 7 (Figure 2A). In the PGE task pyruvate was very efficient, since the time to climb was only $2-3$ s during the whole observation period, whereas $\beta \mathrm{HB}$ was less effective from days 4-7, with climbing times of 4-5 s (Figure 2B).

In contrast to the notable protective effect of pyruvate and $\beta \mathrm{HB}$, neither GEE or ascorbate, alone or in combination, prevented the AMPA-induced motor deficits observed in the rotarod. In these animals the times to fall were similar or even lesser (days 1 and 4) than those shown by the AMPA group during the whole period of observation (Figure 2A). In the PGE test (Figure 2B), GEE was also totally ineffective during all observation period, but ascorbate partially protected, especially during the first three days, where the time to climb was similar to those with pyruvate and $\beta$ HB. However, when ascorbate was co-infused with GEE the protection during the first three days was diminished (Figure 2B), as shown also by the complete paralysis observed in the ipsilateral rear limb in these animals (Figure 1).

\section{Energy substrates reduced the AMPA-induced MN degeneration}

As described in Methods, we analyzed two regions of the lumbar spinal cord (about two lumbar segments for each region). The infused region refers to the area where the cannula was implanted and the rostral region refers to the next rostral segments. We analyzed both regions because we found a differential effect of AMPA depending of its proximity to the probe. This pattern of damage correlates, as explained in the Discussion, with the progression of the bilateral paralysis.

As shown in Figures 3 and 4, the infusion of AMPA resulted at day 7 in an almost total loss of MNs (95\%) in the ipsilateral horn and a reduction of $60 \%$ in the contralateral horn in the infused region, while in the rostral region the degeneration was also notable in both horns but less severe. The infusion of pyruvate and $\beta \mathrm{HB}$ significantly reduced the MNs loss in the infused region to $40 \%-50 \%$ in the ipsilateral horn and to only $10 \%-15 \%$ in the contralateral side. The protection by both substrates was also evident in the rostral region, where the number of healthy MNs was similar to the values in the control rats, especially in the contralateral horn. The
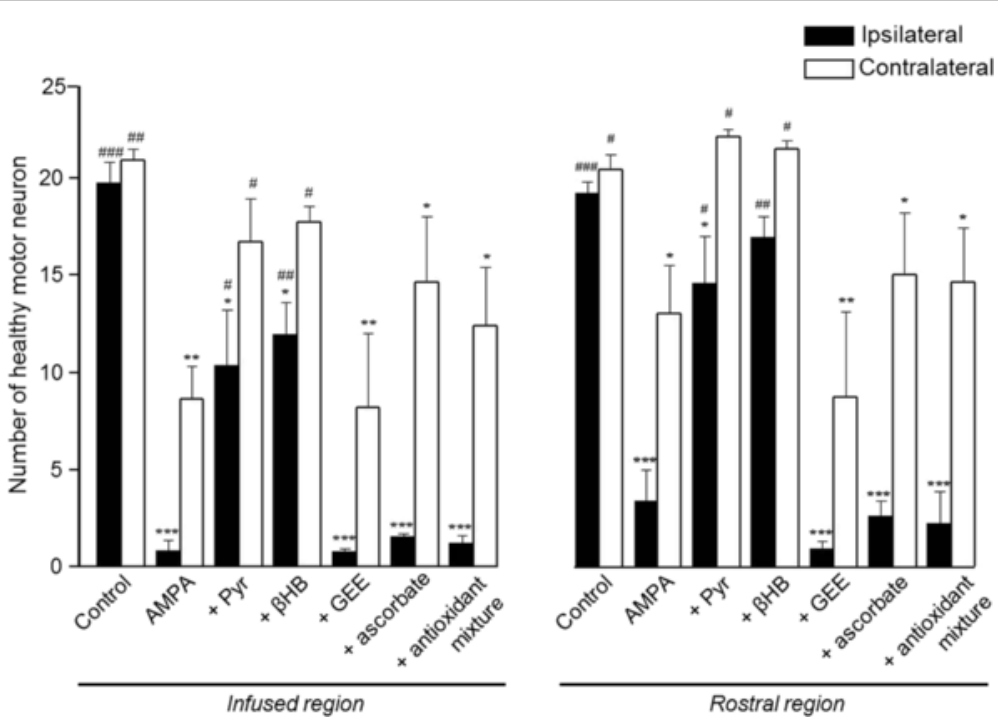

Figure 4 Quantitative analysis of MN loss in each experimental group. Number of healthy MNs in the ipsilateral and contralateral ventral horns in the infused spinal cord region and in the adjacent rostral region of rats treated as indicated, seven days after minipump implantation. Pyruvate and $\beta \mathrm{HB}$ notably prevented the AMPA-induced MN degeneration in the ipsilateral horn in both the infused and rostral regions, whereas ascorbate and GEE, alone or in combination (antioxidant mixture) were ineffective. Fifteen histological slices/rat/region were analyzed. Data are mean values \pm SEM for the following number of rats in each group: control 7; AMPA 16; AMPA + Pyr 10; AMPA + BHB 10; AMPA + GEE 7; AMPA + ascorbate 8; AMPA + antioxidants mixture $8 .{ }^{*} \mathrm{p}<0.05,{ }^{* *} \mathrm{p}<0.01,{ }^{* * *} \mathrm{p}<0.001$ vs the corresponding side of the control group; ${ }^{\#} \mathrm{p}<0.05,{ }^{\# \#} \mathrm{p}<0.01,{ }^{\# \# \#} \mathrm{p}<0.001 \mathrm{vs}$ the corresponding side of the AMPA group. 


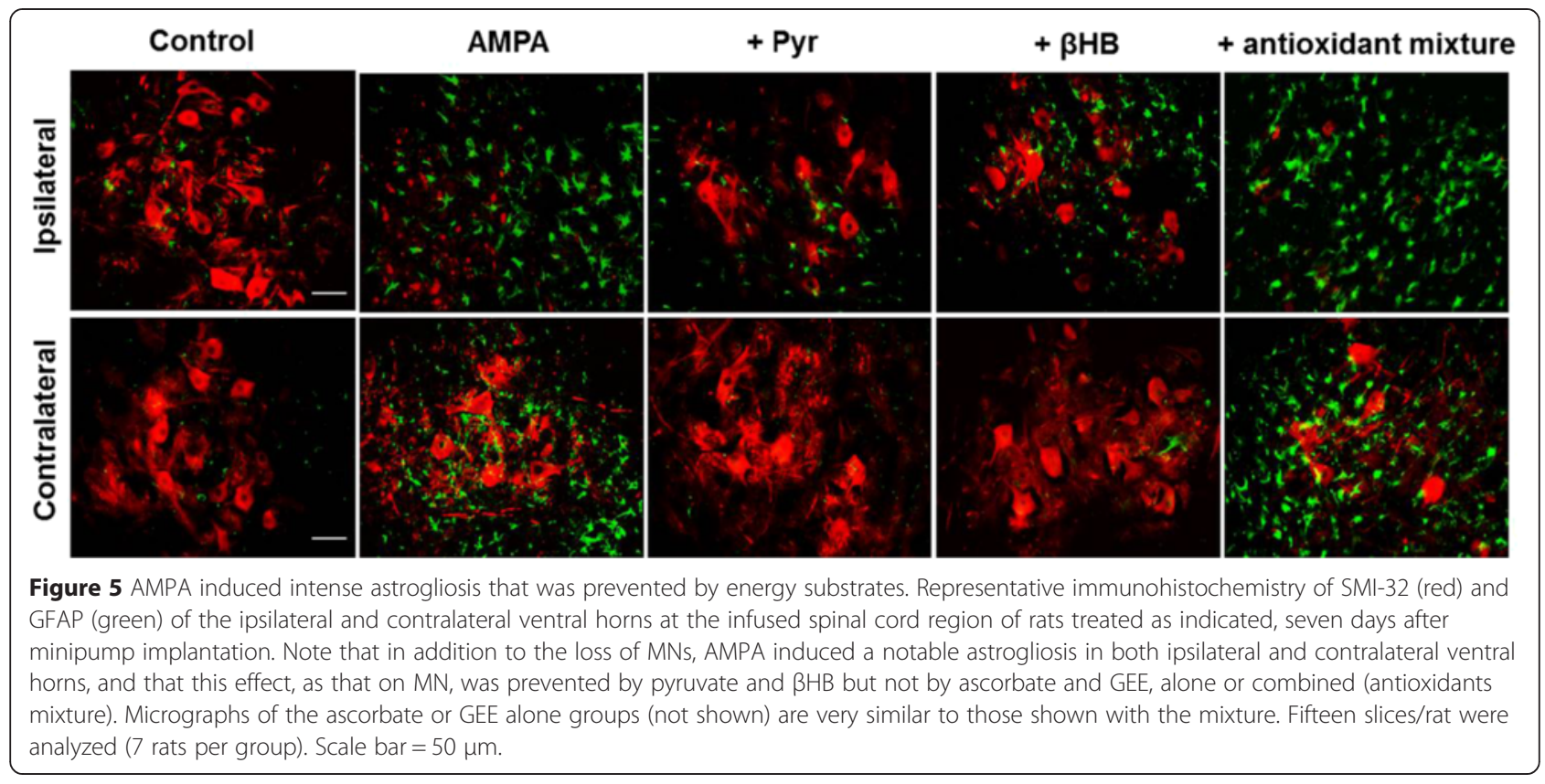

infusion of the antioxidant GEE was ineffective to protect the spinal MNs in both regions, but ascorbate alone or in combination with GEE slightly reduced the number of MNs death, particularly in the contralateral horn of the infused region; these changes, however, were not significant (Figure 4).

Figure 5 shows that the loss of MN produced by AMPA in the infused region was accompanied by a remarkable astrogliosis, as detected by GFAP immunoreactivity, in both the ipsilateral and contralateral horns. Similarly to the protection of MN, both energy substrates tested almost completely prevented the glial reactivity in the contralateral horn and clearly reduced it in the ipsilateral side. In contrast, the GFAP immunoreactivity in the groups treated with AMPA + antioxidants alone or in combination was not different from the AMPA group. These results were reproduced in each of the 7 rats treated in each group of animals and quantitative analysis was not carried out.

\section{Discussion}

In this work we show that the spinal $\mathrm{MN}$ death and astrogliosis induced by the continuous slow infusion of AMPA are prevented by pyruvate and $\beta \mathrm{HB}$, and in one of the motor parameters studied by ascorbate, but not by GEE. Although the protection by the energy substrates was not complete, the number of protected MNs was enough to notably prevent the progression of the AMPA-induced motor deficits. These results show a clear correlation between the progress of motor alterations and significantly reduced $\mathrm{MN}$ loss and astrogliosis.
The AMPA-induced paralysis started in the ipsilateral phalanges and extended with time to the upper regions of the rear limb and to the contralateral side. This suggests that progression of the effects is due to a slow continuous diffusion of AMPA, explaining the larger damage in the infused region as compared with the rostral one. Because the infusion cannula was located between L3 and L4 segments of the spinal cord, the nearest MNs to the probe, that innervate the extensor digitorum that controls the phalanges movement are probably the first ones to receive AMPA and to degenerate, and then AMPA diffuses to affect MNs in the rostral region innervating upper limb muscles. The delay in the appearance of symptoms in the contralateral side is probably due also to the diffusion of the drug.

As mentioned in the Introduction, we have previously reported that pyruvate and $\beta \mathrm{HB}$ protected against the rapid deleterious action of the perfusion of AMPA during $25 \mathrm{~min}$ by reverse microdialysis, which induces unilateral paralysis and $\mathrm{MN}$ death in 3-12 $\mathrm{h}$ [21]. This acute effect allows just a restricted analysis of motor alterations and leaves open the question of whether these energy substrates are effective when the degeneration process is much slower, as it occurs in the osmotic pump continuous infusion procedure used in the present work. A therapeutic effect of oral administration of pyruvate was also reported in the mutant G93A-SOD ALS mice [23], although in these transgenic mice daily i.p. administration of pyruvate failed to delay the onset and motor decline [24]. Pyruvate or $\beta \mathrm{HB}$ also protected spinal MNs from mechanical damage [25], as well as striatal or hippocampal neurons from oxidative stress 
[26] or glutamate-induced excitotoxicity $[27,28]$. However, this is the first report of protection by pyruvate and $\beta \mathrm{HB}$ in a non-genetic model of spinal $\mathrm{MN}$ degeneration that correlated with the progression of motor impairment during several days.

It is noteworthy that there was no significant difference among the protective effect produced by pyruvate and $\beta \mathrm{HB}$ (tested at the same $20 \mathrm{mM}$ concentration), thus suggesting that these substrates share a neuroprotective mechanism. A possible interaction of pyruvate and $\beta \mathrm{HB}$ with AMPA to explain the protection is improbable, because, judging from the structure of these molecules it is difficult to envisage the possibility of such interaction, especially considering that it should be occurring with both substrates and these molecules differ considerably. In addition, in our previous work using microdialysis $[15,21]$, pyruvate and $\beta \mathrm{HB}$ perfusion started 60 min before AMPA and still protected. Furthermore, we have shown that pyruvate and $\beta \mathrm{HB}$ are also good protectors against glutamate-mediated excitotoxicity in the hippocampus, in an in vivo model that does not use AMPA but neurodegeneration is induced by stimulation of glutamate release by 4-aminopyridine [28]. On the other hand, these substrates share an important role in energy metabolism. It is widely known that pyruvate is the end product of glycolysis that can be oxidized by mitochondria driving the synthesis of ATP [29]. Pyruvate can also be taken by astrocytes and released as lactate to serve as energy substrate to neighboring neurons. $\beta \mathrm{HB}$ is a ketone body, a water-soluble mitochondrial fuel, that can be used by neurons when its concentration increases in the nervous system [30,31]. It is probably through these energy-linked mechanisms that pyruvate and $\beta \mathrm{HB}$ protect MN. In support of this interpretation, in cultured neurons these substrates protect against the excitotoxic damage produced by NMDA or glutamate, by stimulating the mitochondrial respiratory capacity and thus improving energy production [32-36], thus suggesting that in vivo they act through such mechanism. However, the protective effect of pyruvate and $\beta \mathrm{HB}$ has also been associated to the ability of $\alpha$-ketoacids to neutralize $\mathrm{H}_{2} \mathrm{O}_{2}$ in a decarboxylating reaction. In cell cultures pyruvate protected neuroblastoma and striatal neurons exposed to $\mathrm{H}_{2} \mathrm{O}_{2}$ by diminishing the production of reactive oxidative species (ROS) $[37,38]$, and $\beta \mathrm{HB}$ protected neuronal cultures exposed to kainate or glutamate also by reducing mitochondrial ROS production [33,34]. Improvement of the mitochondrial antioxidant defenses by MitoQ [39] or by the overexpression of antioxidant enzymes [40] also protected MNs from neurodegeneration induced by mutant G93A SOD1. Nevertheless, our results with ascorbate and GEE, an enzymatic cofactor and potent antioxidant, and one of the major antioxidant defenses of the cell, respectively, show that these compounds were ineffective, except for some improvement in the PGE task with ascorbate alone or in combination with GEE, which however cannot be correlated with a protection against MN loss. Thus, although a role of oxidative stress cannot be completely discarded, especially in the mutant SOD-linked MN degeneration, our results indicate that this mechanism is not the predominant factor in the MN death induced by chronic excitotoxicity, in agreement with our previous results with the acute infusion of AMPA [21].

An important factor in ALS appears to be a non-cell autonomous neuron death in which other cellular types, mainly glial cells, may play a role [41]. In this respect, our results show that AMPA induced a notable reactive astrocytosis that was, similarly to MN loss, prevented by the energy substrates but not by the antioxidants, suggesting that it is a consequence of MNs death rather than a direct effect of AMPA.

\section{Conclusion}

This work shows that mitochondrial energy substrates protect spinal MNs from the neurotoxic cascade triggered by chronic excitotoxicity due to AMPA receptors overactivation, whereas antioxidants were not effective. We conclude that deficits in mitochondrial energy metabolism are an important factor in the mechanisms of this excitotoxic $\mathrm{MN}$ death in the spinal cord, while the role of oxidative stress is less significant. Because glutamate-mediated excitotoxicity has been postulated as a causative factor in $\mathrm{MN}$ degeneration in sporadic ALS, these findings suggest possible preventive or therapeutic strategies for the disease.

\section{Abbreviations}

ALS: Amyotrophic lateral sclerosis; AMPA: a-amino-3-hydroxy-5-methyl-4isoxazole propionate; GEE: Glutathione ethyl ester; GFAP: Glial fibrillary acidic protein; MN: Motor neuron; PGE: Paw grip endurance; ROS: Reactive oxidative species; SOD1: Superoxide dismutase 1; $\beta H B$ : $\beta$-hydroxybutyrate.

\section{Competing interests}

The authors declare that they have no competing interests.

\section{Authors' contributions}

RT designed the research. RT and CN defined the experiments, analyzed the data and drafted the manuscript. CN carried out the experiments. All authors read and approved the final manuscript.

\section{Acknowledgments}

This work was supported by Consejo Nacional de Ciencia y Tecnología (CONACYT, project number 128229) and Dirección General de Asuntos del Personal Académico, Universidad Nacional Autónoma de México (project IN201013). C.N. is recipient of a scholarship from CONACYT. This work is part of the Ph.D. thesis in Biochemical Sciences of C.N.

Received: 21 April 2015 Accepted: 23 April 2015

Published online: 14 May 2015

\section{References}

1. Pasinelli P, Brown RH (2006) Molecular biology of amyotrophic lateral sclerosis: insights from genetics. Nat Rev Neurosci 7:710-723 
2. Santa-Cruz LD, Ramírez-Jarquín UN, Tapia R (2012) Role of mitochondrial dysfunction in motor neuron degeneration in ALS. In: Maurer MH (ed) Amyotrophic Lateral Sclerosis., InTech. doi:10.5772/31290

3. Vielhaber S, Winkler K, Kirches E, Kunz D, Büchner M, Feistner H, Elger CE, Ludolph AC, Riepe MW, Kunz WS (1999) Visualization of defective mitochondrial function in skeletal muscle fibers of patients with sporadic amyotrophic lateral sclerosis. J Neurol Sci 169:133-139

4. Sasaki S, Iwata M (2007) Mitochondrial alterations in the spinal cord of patients with sporadic amyotrophic lateral sclerosis. J Neuropathol Exp Neurol 66:10-16

5. Crugnola V, Lamperti C, Lucchini V, Ronchi D, Peverelli L, Prelle A, Sciacco M, Bordoni A, Fassone E, Fortunato F, Corti S, Silani V, Bresolin N, Di Mauro S, Comi GP, Moggio M (2010) Mitochondrial respiratory chain dysfunction in muscle from patients with amyotrophic lateral sclerosis. Arch Neurol 67:849-854, doi:10.1001/archneurol.2010.128

6. Bernardini C, Censi F, Lattanzi W, Barba M, Calcagnini G, Giuliani A, Tasca G, Sabatelli M, Ricci E, Michetti F (2013) Mitochondrial network genes in the skeletal muscle of amyotrophic lateral sclerosis patients. PLoS One. doi:10.1371/journal.pone.0057739.

7. Bendotti C, Calvaresi N, Chiveri L, Prelle A, Moggio M, Braga M, Silani V, De Biasi S (2001) Early vacuolization and mitochondrial damage in motor neurons of FALS mice are not associated with apoptosis or with changes in cytochrome oxidase histochemical reactivity. J Neurol Sci 191:25-33

8. Parone PA, Da Cruz S, Han JS, McAlonis-Downes M, Vetto AP, Lee SK, Tseng E, Cleveland DW (2013) Enhancing mitochondrial calcium buffering capacity reduces aggregation of misfolded SOD1 and motor neuron cell death without extending survival in mouse models of inherited amyotrophic lateral sclerosis. J Neurosci 33:4657-4671, doi:10.1523/JNEUROSCI.1119-12.2013

9. Mattiazzi M, D’Aurelio M, Gajewski CD, Martushova K, Kiaei M, Beal MF, Manfredi G (2002) Mutated human SOD1 causes dysfunction of oxidative phosphorylation in mitochondria of transgenic mice. J Biol Chem 277:29626-29633

10. Ferri A, Cozzolino M, Crosio C, Nencini M, Casciati A, Gralla EB, Rotilio G, Valentine JS, Carrì MT (2006) Familial ALS-superoxide dismutases associate with mitochondria and shift their redox potentials. Proc Natl Acad Sci U S A 103:13860-13865

11. Shaw PJ (1999) Calcium, glutamate, and amyotrophic lateral sclerosis: more evidence but no certainties. Ann Neurol 46:803-805

12. Grosskreutz J, Van Den Bosch L, Keller BU (2010) Calcium dysregulation in amyotrophic lateral sclerosis. Cell Calcium 47:165-174, doi:10.1016/ j.ceca.2009.12.002

13. Wang Y, Qin ZH (2010) Molecular and cellular mechanisms of excitotoxic neuronal death. Apoptosis 15:1382-1402, doi:10.1007/s10495-010-0481-0

14. Corona JC, Tapia R (2004) AMPA receptor activation, but not the accumulation of endogenous extracellular glutamate, induces paralysis and motor neuron death in rat spinal cord in vivo. J Neurochem 89:988-997

15. Corona JC, Tapia R (2007) $\mathrm{Ca}^{2+}$-permeable AMPA receptors and intracellular $\mathrm{Ca}^{2+}$ determine motoneuron vulnerability in rat spinal cord in vivo. Neuropharmacology 52:1219-1228

16. Tovar-y-Romo LB, Zepeda A, Tapia R (2007) Vascular endothelial growth factor prevents paralysis and motoneuron death in a rat model of excitotoxic spinal cord neurodegeneration. J Neuropathol Exp Neurol 66:913-922

17. Corona JC, Tapia R (2008) Calpain inhibition protects spinal motoneurons from the excitotoxic effects of AMPA in vivo. Neurochem Res 33:1428-1434, doi:10.1007/s1 1064-007-9559-7

18. Carriedo SG, Yin HZ, Weiss JH (1996) Motor neurons are selectively vulnerable to AMPA/kainate receptor-mediated injury in vitro. J Neurosci 16:4069-4079

19. Carriedo SG, Sensi SL, Yin HZ, Weiss JH (2000) AMPA exposures induce mitochondrial $\mathrm{Ca}(2+)$ overload and ROS generation in spinal motor neurons in vitro. J Neurosci 20:240-250

20. Weiss $\mathrm{JH}$, Sensi SL (2000) $\mathrm{Ca}^{2+}-\mathrm{Zn}^{2+}$ permeable AMPA or kainate receptors: possible key factors in selective neurodegeneration. Trends Neurosci 23:365-371

21. Santa-Cruz LD, Tapia R (2014) Role of energy metabolic deficits and oxidative stress in excitotoxic spinal motor neuron degeneration in vivo. ASN Neuro. doi:10.1042/AN20130046.

22. Zeevalk GD, Manzino L, Sonsalla PK, Bernard LP (2007) Characterization of intracellular elevation of glutathione (GSH) with glutathione monoethyl ester and GSH in brain and neuronal cultures: relevance to Parkinson's disease. Exp Neurol 203:512-520
23. Ari C, Poff AM, Held HE, Landon CS, Goldhagen CR, Mavromates N, D'Agostino DP (2014) Metabolic therapy with Deanna protocol supplementation delays disease progression and extends survival in amyotrophic lateral sclerosis (ALS) mouse model. PLoS One. doi:10.1371/ journal.pone.0103526.

24. Esposito E, Capasso M, di Tomasso N, Corona C, Pellegrini F, Uncini A, Vitaglione P, Fogliano V, Piantelli M, Sensi SL (2007) Antioxidant strategies based on tomato-enriched food or pyruvate do not affect disease onset and survival in an animal model of amyotrophic lateral sclerosis. Brain Res 1168:90-96

25. Yuan Y, Su Z, Pu Y, Liu X, Chen J, Zhu F, Zhu Y, Zhang H, He C (2012) Ethyl pyruvate promotes spinal cord repair by ameliorating the glial microenvironment. Br J Pharmacol 166:749-763, doi:10.1111/j.14765381.2011.01804.x

26. Satpute R, Lomash V, Kaushal M, Bhattacharya R (2013) Neuroprotective effects of a-ketoglutarate and ethyl pyruvate against motor dysfunction and oxidative changes caused by repeated 1-methyl-4-phenyl-1,2,3,6 tetrahydropyridine exposure in mice. Hum Exp Toxicol 32:747-758, doi:10.1177/0960327112468172

27. Mejía-Toiber J, Montiel T, Massieu L (2006) D-beta-hydroxybutyrate prevents glutamate-mediated lipoperoxidation and neuronal damage elicited during glycolysis inhibition in vivo. Neurochem Res 31:1399-1408

28. Netzahualcoyotzi C, Tapia R (2014) Energy substrates protect hippocampus against endogenous glutamate-mediated neurodegeneration in awake rats. Neurochem Res 39:1346-1354, doi:10.1007/s11064-014-1318-y

29. Nicholls DG, Budd SL (2000) Mitochondria and neuronal survival. Physiol Rev 80:315-360

30. Fernandez-Fernandez S, Almeida A, Bolaños JP (2012) Antioxidant and bioenergetics coupling between neurons and astrocytes. Biochem J 443:3-11, doi:10.1042/BJ20111943

31. Dienel GA (2013) Astrocytic energetics during excitatory neurotransmission: What are contributions of glutamate oxidation and glycolysis? Neurochem Int 63:244-258, doi:10.1016/j.neuint.2013.06.015

32. Maus M, Marin P, Israël M, Glowinski J, Prémont J (1999) Pyruvate and lactate protect striatal neurons against N-methyl-D-aspartate-induced neurotoxicity. Eur J Neurosci 11:3215-3224

33. Noh HS, Hah YS, Nilufar R, Han J, Bong JH, Kang SS, Cho GJ, Choi WS (2006) Acetoacetate protects neuronal cells from oxidative glutamate toxicity. J Neurosci Res 83:702-709

34. Maalouf M, Sullivan PG, Davis L, Kim DY, Rho JM (2007) Ketones inhibit mitochondrial production of reactive oxygen species production following glutamate excitotoxicity by increasing NADH oxidation. Neuroscience 145:256-264

35. Izumi Y, Zorumski CF (2010) Neuroprotective effects of pyruvate following NMDA-mediated excitotoxic insults in hippocampal slices. Neurosci Lett 478:131-135, doi:10.1016/j.neulet.2010.04.078

36. Laird MD, Clerc P, Polster BM, Fiskum G (2013) Augmentation of normal and glutamate-impaired neuronal respiratory capacity by exogenous alternative biofuels. Transl Stroke Res 4:643-651, doi:10.1007/s12975-013-0275-0

37. Desagher S, Glowinski J, Prémont J (1997) Pyruvate protects neurons against hydrogen peroxide-induced toxicity. J Neurosci 17:9060-9067

38. Wang X, Perez E, Liu R, Yan LJ, Mallet RT, Yang SH (2007) Pyruvate protects mitochondria from oxidative stress in human neuroblastoma SK-N-SH cells. Brain Res 1132:1-9

39. Miquel E, Cassina A, Martínez-Palma L, Souza JM, Bolatto C, Rodríquez-Bottero S, Logan A, Smith RA, Murphy MP, Barbeito L, Radi R, Cassina P (2014) Neuroprotective effects of the mitochondria-targeted antioxidant MitoQ in a model of inherited amyotrophic lateral sclerosis. Free Radic Biol Med 70:204-213, doi:10.1016/j.freeradbiomed.2014.02.019

40. Liu R, Li B, Flanagan SW, Oberley LW, Gozal D, Qiu M (2002) Increased mitochondrial antioxidative activity or decreased oxygen free radical propagation prevent mutant SOD1-mediated motor neuron cell death and increase amyotrophic lateral sclerosis-like transgenic mouse survival. J Neurochem 80:488-500

41. Philips T, Rothstein JD (2014) Glial cells in amyotrophic lateral sclerosis. Exp Neurol. doi:10.1016/j.expneurol.2014.05.015. 